\title{
Assessment of the elderly's functional ability to manage their medication: a systematic literature review
}

International Journal of Clinical Pharmacy

pp 1-15

\section{Authors Authors and affiliations}

Ana Margarida Advinha, Manuel José Lopes, Sofia de Oliveira-Martins

Review Article

First Online: 10 December 2016

DOI (Digital Object Identifier): 10.1007/s11096-016-0409-z

Cite this article as:

Advinha, A.M., Lopes, M.J. \& de Oliveira-Martins, S. Int J Clin Pharm (2016).

doi:10.1007/s11096-016-0409-z

\begin{abstract}
Background The evaluation of the elderly's ability to manage medication through the use of a validated tool can be a significant step in identifying inabilities and needs, with the objective of increasing their self-care skills, and promoting successful aging. Aim of the review To identify studies assessing the elderly's functional ability to manage their own medication. Method For the search strategy, the PICO method was used: P-Population (elderly), I-Instruments (tools for assessing medication management ability), C-Context (community) and $\mathrm{O}-$ Outcomes (functional ability to manage medication). The final search query was run in MEDLINE/PubMed, CINAHL Plus, ISI Web of Science and Scopus. The whole process was developed according to the PRISMA statement. Results The search retrieved 8051 records. In each screening stage, the selection criteria were applied to eliminate records where at least one of the exclusion criteria was verified. At the end of this selection, we obtained a total of 18 papers (17 studies). The results allow the conclusion to be drawn that studies use several different instruments, most of them not validated. The authors agree that medication management abilities decrease as cognitive impairment increases, even if a lot of studies assess only the physical dimension. DRUGS was the instrument most often used. Conclusion Older adults' ability to manage their medication should be assessed using tools specifically built and validate for the purpose. DRUGS (which uses the real regimen taken by the elderly) was the most widely used assessment instrument in the screened studies.
\end{abstract}

Keywords 
Aged Functional ability Geriatric assessment Medication

management Systematic review

\section{References}

1. Maddigan SL, Farris KB, Keating N, Wiens CA, Johnson JA. Predictors of older adults' capacity for medication management in a self-medication program: a retrospective chart review. J Aging Health. 2003;15:332-52.

CrossRef (http://dx.doi.org/10.1177/o898264303251893)

PubMed (http://www.ncbi.nlm.nih.gov/entrez/query.fcgi? $\mathrm{cmd}=$ Retrieve\&db=PubMed\&dopt=Abstract\&list_uids=12795276)

Google Scholar (http://scholar.google.com/scholar_lookup?title=Predictors\%20of\%20older\%20adults\%E2\%80\%99\%2 medication\%20program\%3A\%20a\%20retrospective\%20chart\%20review\&author=SL.\%2OMaddigan\&author=KB.\%20Fa $352 \&$ publication_year $=2003$ )

2. World Health Organization. Ageing and health technical report. Japan: Kobe; 2004.

Google Scholar (http://scholar.google.com/scholar_lookup?

title=Ageing\%20and\%2ohealth\%2otechnical\%2oreport\&publication_year=2004）

3. Tordoff J, Simonsen K, Thomson WM, Norris PT. “It's just routine”. A qualitative study of medicine-taking amongst older people in New Zealand. Pharm World Sci.

2010;32:154-61.

CrossRef (http://dx.doi.org/10.1007/s11096-009-9361-5)

PubMed (http://www.ncbi.nlm.nih.gov/entrez/query.fcgi?

$\mathrm{cmd}=$ Retrieve $\& \mathrm{db}=$ PubMed\&dopt=Abstract\&list_uids=20043241)

Google Scholar (http://scholar.google.com/scholar_lookup?title=\%E2\%80\%9CIt\%E2\%80\%99s\%20just\%20routine\%Ec taking\%20amongst\%20older\%2opeople\%20in\%20New\%20Zealand\&author=J.\%20Tordoff\&author=K.\%20Simonsen\&a 161\&publication_year=2010)

4. MacLaughlin EJ, Raehl CL, Treadway AK, Sterling TL, Zoller DP, Bond CA.

Assessing medication adherence in the elderly-which tools to use in clinical practice? Drugs Aging. 2005;22(3):231-55.

CrossRef (http://dx.doi.org/10.2165/00002512-200522030-00005)

PubMed (http://www.ncbi.nlm.nih.gov/entrez/query.fcgi?

$\mathrm{cmd}=$ Retrieve\&db=PubMed\&dopt=Abstract\&list_uids=15813656)

Google Scholar (http://scholar.google.com/scholar_lookup?

title=Assessing\%20medication\%20adherence\%20in\%20the\%2oelderly\%E2\%80\%94which\%20tools\%20to\%20use\%2oin 255\&publication_year $=2005$ )

5. Patterson TL, Lacro J, McKibbin CL, Moscona S, Hughs T, Jeste DV. Medication management ability assessment: results from a performance-based measure in older outpatients with schizophrenia. J Clin Psychopharmacol. 2002;22(1):11-9.

CrossRef (http://dx.doi.org/10.1097/00004714-200202000-00003)

PubMed (http://www.ncbi.nlm.nih.gov/entrez/query.fcgi? cmd=Retrieve\&db=PubMed\&dopt=Abstract\&list_uids=11799337)

Google Scholar (http://scholar.google.com/scholar_lookup?title=Medication\%2omanagement\%20ability\%20assessmer based\%20measure\%20in\%2oolder\%20outpatients\%20with\%20schizophrenia\&author=TL.\%2oPatterson\&author=J.\%2( 19\&publication_year=2002)

6. Elliott RA, Marriott JL. Standardised assessment of patients' capacity to manage medications: a systematic review of published instruments. BMC Geriatr.

2009;9:27.

CrossRef (http://dx.doi.org/10.1186/1471-2318-9-27)

PubMed (http://www.ncbi.nlm.nih.gov/entrez/query.fcgi? 
cmd=Retrieve\&db=PubMed\&dopt=Abstract\&list_uids=19594913)

PubMedCentral (http://www.ncbi.nlm.nih.gov/pmc/articles/PMC2719637)

Google Scholar (http://scholar.google.com/scholar_lookup?

title=Standardised\%20assessment\%20of\%2opatients\%E2\%80\%99\%2ocapacity\%20to\%2omanage\%2omedications\%3A\%

7. Farris KB, Phillips BB. Instruments assessing capacity to manage medications. Ann

Pharmacother. 2008;42:1026-36.

CrossRef (http://dx.doi.org/10.1345/aph.1G502)

PubMed (http://www.ncbi.nlm.nih.gov/entrez/query.fcgi?

cmd=Retrieve\&db=PubMed\&dopt=Abstract\&list_uids=18594054)

Google Scholar (http://scholar.google.com/scholar_lookup?

title=Instruments\%20assessing\%20capacity\%20to\%20manage\%20medications\&author=KB.\%20Farris\&author=BB.\%2( 1036\&publication_year=2008)

8. Kucukarslan SN, Hagan AM, Shimp LA, Gaither CA, Lewis NJ. Integrating

medication therapy management in the primary care medical home: a review of

randomized controlled trials. Am J Health Syst Pharm. 2011;68(328):335-45.

CrossRef (http://dx.doi.org/10.2146/ajhp100405)

PubMed (http://www.ncbi.nlm.nih.gov/entrez/query.fcgi?

$\mathrm{cmd}=$ Retrieve\&db=PubMed\&dopt=Abstract\&list_uids=21289329)

Google Scholar (http://scholar.google.com/scholar_lookup?

title=Integrating\%2omedication\%2otherapy\%2omanagement\%20in\%2othe\%2oprimary\%2ocare\%2omedical\%2ohome;

345\&publication_year=2011)

9. Irvine-Meek J, Gould ON, Wheaton H, Todd LE. Acceptability and face validity of a

geriatric self-medication assessment tool. Can J Hosp Pharm. 2010;63(3):225-32.

PubMed (http://www.ncbi.nlm.nih.gov/entrez/query.fcgi?

$\mathrm{cmd}=$ Retrieve\&db=PubMed\&dopt=Abstract\&list_uids=22478982)

PubMedCentral (http://www.ncbi.nlm.nih.gov/pmc/articles/PMC2901782)

Google Scholar (http://scholar.google.com/scholar_lookup?title=Acceptability\%20and\%2oface\%2ovalidity\%20of\%20a medication\%20assessment\%20tool\&author=J.\%20Irvine-

Meek\&author=ON.\%20Gould\&author=H.\%20Wheaton\&author=LE.\%20Todd\&journal=Can\%20J\%20Hosp\%20Pharm\& 232\&publication_year=2010)

10. Irvine-Meek JM, Gould ON. Psychometric evaluation of a self-medication

assessment tool in an elderly population. Can J Hosp Pharm. 2011;64(1):16-24.

PubMed (http://www.ncbi.nlm.nih.gov/entrez/query.fcgi?

cmd=Retrieve\&db=PubMed\&dopt=Abstract\&list_uids=22479024)

PubMedCentral (http://www.ncbi.nlm.nih.gov/pmc/articles/PMC3053188)

Google Scholar (http://scholar.google.com/scholar_lookup?

title=Psychometric\%2oevaluation\%20of\%20a\%20self-

medication\%20assessment\%20tool\%20in\%20an\%2oelderly\%2opopulation\&author=JM.\%2oIrvine-

Meek\&author=ON.\%20Gould\&journal=Can\%20J\%20Hosp\%20Pharm\&volume=64\&issue=1\&pages=16-

24\&publication_year=2011)

11. Orwig D, Brandt N, Gruber-Baldini AL. Medication management assessment for

older adults in the community. Gerontologist. 2006;46:661-8.

CrossRef (http://dx.doi.org/10.1093/geront/46.5.661)

PubMed (http://www.ncbi.nlm.nih.gov/entrez/query.fcgi?

cmd=Retrieve\&db=PubMed\&dopt=Abstract\&list_uids=17050757)

Google Scholar (http://scholar.google.com/scholar_lookup?

title=Medication\%2omanagement\%20assessment\%20for\%20older\%20adults\%20in\%20the\%2ocommunity\&author=D.\%

Baldini\&journal $=$ Gerontologist\&volume $=46 \&$ pages $=661-668 \&$ publication $\_$year $=2006$ )

12. Carlson MC, Fried LP, Xue QL, Tekwe C, Brandt J. Validation of the Hopkins

Medication Schedule to identify difficulties in taking medications. J Gerontol A Biol 
Sci Med Sci. 2005;60:217-23.

CrossRef (http://dx.doi.org/10.1093/gerona/60.2.217)

PubMed (http://www.ncbi.nlm.nih.gov/entrez/query.fcgi?

$\mathrm{cmd}=$ Retrieve\&db=PubMed\&dopt=Abstract\&list_uids=15814866)

Google Scholar (http://scholar.google.com/scholar_lookup?

title=Validation\%20of\%2othe\%20Hopkins\%20Medication\%20Schedule\%2oto\%2oidentify\%2odifficulties\%2oin\%20taki 223\&publication_year $=2005$ )

13. Schmidt KS, Lieto JM. Validity of the Medication Administration Test among older adults with and without dementia. Am J Geriatr Pharmacother. 2005;3:255-61.

CrossRef (http://dx.doi.org/10.1016/j.amjopharm.2005.12.003)

PubMed (http://www.ncbi.nlm.nih.gov/entrez/query.fcgi?

$\mathrm{cmd}=$ Retrieve\&db=PubMed\&dopt=Abstract\&list_uids=16503321)

Google Scholar (http://scholar.google.com/scholar_lookup?

title=Validity\%20of\%2othe\%20Medication\%20Administration\%20Test\%20among\%20older\%20adults\%20with\%20and $261 \&$ publication_year $=2005$ )

14. Beckman AGK, Parker MG, Thorslund M. Can elderly people take their medicine?

Patient Educ Couns. 2005;59:186-91.

CrossRef (http://dx.doi.org/10.1016/j.pec.2004.11.005)

PubMed (http://www.ncbi.nlm.nih.gov/entrez/query.fcgi?

cmd=Retrieve\&db=PubMed\&dopt=Abstract\&list_uids=16257624)

Google Scholar (http://scholar.google.com/scholar_lookup?

title=Can\%2oelderly\%2opeople\%2otake\%2otheir\%2omedicine\%3F\&author=AGK.\%2oBeckman\&author=MG.\%20Park€ $191 \&$ publication_year=2005)

15. Heaton RK, Marcotte TD, Mindt MR, Sadek J, Moore DJ, Bentley H, et al. The impact of HIV-associated neuropsychological impairment on everyday functioning.

J Int Neuropsychol Soc. 2004;10(3):317-31.

CrossRef (http://dx.doi.org/10.1017/S1355617704102130)

PubMed (http://www.ncbi.nlm.nih.gov/entrez/query.fcgi?

cmd=Retrieve\&db=PubMed\&dopt=Abstract\&list_uids=15147590)

Google Scholar (http://scholar.google.com/scholar_lookup?title=The\%20impact\%20of\%2oHIV-

associated\%20neuropsychological\%20impairment\%20on\%20everyday\%2ofunctioning\&author=RK.\%20Heaton\&author 331\&publication_year=2004)

16. Raehl CL, Bond CA, Woods T, Patry RA, Sleeper RB. Individualized drug use assessment in the elderly. Pharmacotherapy. 2002;22:1239-48.

CrossRef (http://dx.doi.org/10.1592/phco.22.15.1239.33473)

PubMed (http://www.ncbi.nlm.nih.gov/entrez/query.fcgi? $\mathrm{cmd}=$ Retrieve\&db=PubMed\&dopt=Abstract\&list_uids=12389875)

Google Scholar (http://scholar.google.com/scholar_lookup?

title=Individualized\%2odrug\%20use\%20assessment\%2oin\%2othe\%2oelderly\&author=CL.\%2oRaehl\&author=CA.\%20B 1248\&publication_year=2002)

17. Edelberg HK, Shallenberger E, Wei JY. Medication management capacity in highly functioning community-living older adults: detection of early deficits. J Am Geriatr

Soc. 1999;47:592-6.

CrossRef (http://dx.doi.org/10.1111/j.1532-5415.1999.tb02574.x)

PubMed (http://www.ncbi.nlm.nih.gov/entrez/query.fcgi?

$\mathrm{cmd}=$ Retrieve\&db=PubMed\&dopt=Abstract\&list_uids=10323653)

Google Scholar (http://scholar.google.com/scholar_lookup?title=Medication\%2omanagement\%20capacity\%20in\%2oh living\%20older\%20adults\%3A\%20detection\%20of\%20early\%20deficits\&author=HK.\%20Edelberg\&author=E.\%20Shall 596\&publication_year=1999)

18. Albert SM, Weber CM, Todak G, Polanco C, Clouse R, McElhiney M, et al. An

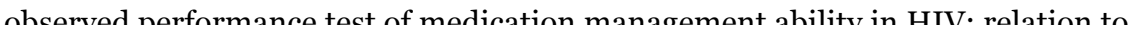




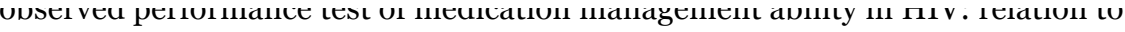

neuropsychological status and medication adherence outcomes. AIDS Behav.

1999;3(2):121-8.

CrossRef (http://dx.doi.org/10.1023/A\%3A1025483806464)

Google Scholar (http://scholar.google.com/scholar_lookup?

title=An\%20observed\%2operformance\%2otest\%20of\%2omedication\%2Omanagement\%2Oability\%20in\%2OHIV\%3A\%2 128\&publication_year=1999)

19. Gurland BJ, Cross P, Chen JM, Wilder DE, Pine ZM, Lantigua RA, et al. A new performance-test of adaptive cognitive-functioning-the Medication Management (MM) Test. Int J Geriatr Psychiatry. 1994;9(11):875-85.

CrossRef (http://dx.doi.org/10.1002/gps.930091104)

Google Scholar (http://scholar.google.com/scholar_lookup?title=A\%20new\%2operformance-test\%20of\%20adaptive\%2 functioning\%E2\%80\%94the\%20Medication\%2oManagement\%20\%28MM\%29\%20Test\&author=BJ.\%20Gurland\&authc 885\&publication_year=1994)

20. Isaac LM, Tamblyn RM. Compliance and cognitive function: a methodological approach to measuring unintentional errors in medication compliance in the elderly. McGill-Calgary Drug Research Team. Gerontologist. 1993;33(6):772-81.

CrossRef (http://dx.doi.org/10.1093/geront/33.6.772)

PubMed (http://www.ncbi.nlm.nih.gov/entrez/query.fcgi?

cmd=Retrieve $\& \mathrm{db}=$ PubMed\&dopt=Abstract\&list_uids=8314104)

Google Scholar (http://scholar.google.com/scholar_lookup?

title=Compliance\%20and\%20cognitive\%2ofunction\%3A\%20a\%2omethodological\%20approach\%20to\%20measuring\%2 Calgary\%20Drug\%20Research\%20Team\&author=LM.\%2oIsaac\&author=RM.\%20Tamblyn\&journal=Gerontologist\&vol

21. Romonko L, Pereles L. An evaluation of pharmacy assessment for geriatric patients.

Can J Hosp Pharm. 1992;45(1):15-20.

PubMed (http://www.ncbi.nlm.nih.gov/entrez/query.fcgi?

$\mathrm{cmd}=$ Retrieve\&db=PubMed\&dopt=Abstract\&list_uids=10117358)

Google Scholar (http://scholar.google.com/scholar_lookup?

title=An\%20evaluation\%20of\%20pharmacy\%20assessment\%2ofor\%2ogeriatric\%2opatients\&author=L.\%2oRomonko\&: 20\&publication_year=1992)

22. Meyer ME, Schuna AA. Assessment of geriatric patients' functional ability to take medication. DICP. 1989;23:171-4.

PubMed (http://www.ncbi.nlm.nih.gov/entrez/query.fcgi?

$\mathrm{cmd}=$ Retrieve\&db=PubMed\&dopt=Abstract\&list_uids=2728508)

Google Scholar (http://scholar.google.com/scholar_lookup?

title=Assessment\%20of\%20geriatric\%2opatients\%E2\%80\%99\%2ofunctional\%20ability\%2oto\%2otake\%2omedication\& 174\&publication_year=1989)

23. Murray MD, Darnell J, Weinberger M, Martz BL. Factors contributing to medication noncompliance in elderly public housing tenants. DICP.

1986;20(2):146-52.

Google Scholar (http://scholar.google.com/scholar_lookup?

title=Factors\%20contributing\%20to\%20medication\%20noncompliance\%20in\%2oelderly\%2opublic\%2ohousing\%20ten 152\&publication_year=1986)

24. Holly C, Salmond SW, Saimbert MK. Comprehensive systematic review for advanced nursing practice. Berlin: Springer; 2011.

Google Scholar (http://scholar.google.com/scholar_lookup?

title=Comprehensive\%2osystematic\%2oreview\%2ofor\%2oadvanced\%2onursing\%2opractice\&author=C.\%20Holly\&auth

25. Liberati A, Altman DG, Tetzlaff J, Mulrow C, Gøtzsche PC, Ioannidis JPA, et al. The PRISMA statement for reporting systematic reviews and meta-analyses of studies that evaluate health care interventions: explanation and elaboration. Ann Intern 
Med. 2009;151:W65-94.

CrossRef (http://dx.doi.org/10.7326/0003-4819-151-4-200908180-00136)

PubMed (http://www.ncbi.nlm.nih.gov/entrez/query.fcgi?

$\mathrm{cmd}=$ Retrieve\&db=PubMed\&dopt=Abstract\&list_uids=19622512)

Google Scholar (http://scholar.google.com/scholar_lookup?title=The\%20PRISMA\%20statement\%20for\%20reporting\% analyses\%20of\%20studies\%20that\%20evaluate\%2ohealth\%20care\%2ointerventions\%3A\%20explanation\%20and\%20el. W94\&publication_year=2009)

26. World Health Organization. Definition of an older or elderly person. 2015.

http://www.who.int/healthinfo/survey/ageingdefnolder/en/

(http://www.who.int/healthinfo/survey/ageingdefnolder/en/). Accessed 1 Oct

2015 .

27. Edelberg HK, Shallenberger E, Hausdorff JM, Wei JY. One-year follow-up of medication management capacity in highly functioning older adults. J Gerontol A

Biol Sci Med Sci. 2000;55:M550-3.

CrossRef (http://dx.doi.org/10.1093/gerona/55.10.M550)

PubMed (http://www.ncbi.nlm.nih.gov/entrez/query.fcgi?

$\mathrm{cmd}=$ Retrieve\&db=PubMed\&dopt=Abstract\&list_uids=11034226)

Google Scholar (http://scholar.google.com/scholar_lookup?title=One-year\%2ofollow-

up\%20of\%2omedication\%2omanagement\%20capacity\%2oin\%2ohighly\%2ofunctioning\%2oolder\%2Oadults\&author=H] M553\&publication_year=2000)

28. Beckman A, Bernsten C, Parker MG, Thorslund M, Fastbom J. The difficulty of opening medicine containers in old age: a population-based study. Pharm World

Sci. 2005;27:393-8.

CrossRef (http://dx.doi.org/10.1007/s11096-005-7903-z)

PubMed (http://www.ncbi.nlm.nih.gov/entrez/query.fcgi?

$\mathrm{cmd}=$ Retrieve\&db=PubMed\&dopt=Abstract\&list_uids=16341746)

Google Scholar (http://scholar.google.com/scholar_lookup?title=The\%2odifficulty\%20of\%2oopening\%2omedicine\%2C based\%20study\&author=A.\%20Beckman\&author=C.\%20Bernsten\&author=MG.\%2OParker\&author=M.\%2OThorslund\& 398\&publication_year=2005)

29. Ruscin JM, Semla TP. Assessment of medication management skills in older outpatients. Ann Pharmacother. 1996;30:1083-8.

PubMed (http://www.ncbi.nlm.nih.gov/entrez/query.fcgi?

cmd=Retrieve\&db=PubMed\&dopt=Abstract\&list_uids=8893112)

Google Scholar (http://scholar.google.com/scholar_lookup?

title=Assessment\%20of\%20medication\%20management\%20skills\%20in\%2oolder\%20outpatients\&author=JM.\%2oRuS 1088\&publication_year=1996)

30. Hutchison LC, Jones SK, West DS, Wei JY. Assessment of medication management by community-living elderly persons with two standardized assessment tools: a cross-sectional study. Am J Geriatr Pharmacother. 2006;4:144-53.

CrossRef (http://dx.doi.org/10.1016/j.amjopharm.2006.06.009)

PubMed (http://www.ncbi.nlm.nih.gov/entrez/query.fcgi?

$\mathrm{cmd}=$ Retrieve\&db=PubMed\&dopt=Abstract\&list_uids=16860261)

Google Scholar (http://scholar.google.com/scholar_lookup?title=Assessment\%20of\%2omedication\%2omanagement\%ء living\%20elderly\%2opersons\%20with\%20two\%2ostandardized\%20assessment\%20tools\%3A\%20a\%20crosssectional\%2Ostudy\&author=LC.\%20Hutchison\&author=SK.\%20Jones\&author=DS.\%20West\&author=JY.\%20Wei\&jour 153\&publication_year=2006)

31. Cotrell V, Wild K, Bader T. Medication management and adherence among cognitively impaired older adults. J Gerontol Soc Work. 2006;47:31-46.

CrossRef (http://dx.doi.org/10.1300/Jo83v47no3_03)

PubMed (http://www.ncbi.nlm.nih.gov/entrez/query.fcgi? 


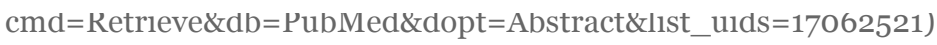

Google Scholar (http://scholar.google.com/scholar_lookup?

title=Medication\%2omanagement\%20and\%20adherence\%20among\%2ocognitively\%2oimpaired\%20older\%20adults\&a 46\&publication_year=2006)

32. Farris KD, Kelly MW, Tryon J. Clock drawing test and medication complexity index

as indicators of medication management capacity: a pilot study. J Am Pharm Assoc.

2003;43:78-81.

CrossRef (http://dx.doi.org/10.1331/10865800360467097)

Google Scholar (http://scholar.google.com/scholar_lookup?

title=Clock\%20drawing\%20test\%20and\%20medication\%20complexity\%2oindex\%20as\%2oindicators\%20of\%20medica 81\&publication_year $=2003$ )

33. Kripalani S, Henderson LE, Chiu EY, Robertson R, Kolm P, Jacobson TA.

Predictors of medication self-management skill in a low-literacy population. J Gen

Intern Med. 2006;21:852-6.

CrossRef (http://dx.doi.org/10.1111/j.1525-1497.2006.00536.x)

PubMed (http://www.ncbi.nlm.nih.gov/entrez/query.fcgi?

$\mathrm{cmd}=$ Retrieve $\& \mathrm{db}=$ PubMed\&dopt=Abstract\&list_uids=16881946)

PubMedCentral (http://www.ncbi.nlm.nih.gov/pmc/articles/PMC1831567)

Google Scholar (http://scholar.google.com/scholar_lookup?title=Predictors\%20of\%2omedication\%20self-management literacy\%20population\&author=S.\%20Kripalani\&author=LE.\%20Henderson\&author=EY.\%20Chiu\&author=R.\%20Rob€ 856\&publication_year=2006)

34. Lakey SL, Gray SL, Borson S. Assessment of older adults' knowledge of and preferences for medication management tools and support systems. Ann

Pharmacother. 2009;43:1011-9.

CrossRef (http://dx.doi.org/10.1345/aph.1L704)

PubMed (http://www.ncbi.nlm.nih.gov/entrez/query.fcgi?

$\mathrm{cmd}=$ Retrieve\&db=PubMed\&dopt=Abstract\&list_uids=19470855)

Google Scholar (http://scholar.google.com/scholar_lookup?

title=Assessment\%20of\%20older\%20adults\%E2\%80\%99\%2oknowledge\%20of\%20and\%2opreferences\%20for\%20medi 1019\&publication_year=2009)

35. Lubinga SJ, Millar I, Babigumira JB. Pilot evaluation of the psychometric properties of a self-medication Risk Assessment Tool among elderly patients in a community setting. BMC Res Notes. 2011;4:398.

CrossRef (http://dx.doi.org/10.1186/1756-0500-4-398)

PubMed (http://www.ncbi.nlm.nih.gov/entrez/query.fcgi?

cmd=Retrieve\&db=PubMed\&dopt=Abstract\&list_uids=21989334)

PubMedCentral (http://www.ncbi.nlm.nih.gov/pmc/articles/PMC3199267)

Google Scholar (http://scholar.google.com/scholar_lookup?title=Pilot\%2oevaluation\%20of\%20the\%2opsychometric\%: medication\%20Risk\%20Assessment\%20Tool\%20among\%20elderly\%2opatients\%20in\%20a\%20community\%20setting $\mathcal{E}$

36. McDougall GJ, Becker H, Vaughan PW, Acee TW, Delville CL. The revised direct assessment of functional status for independent older adults. Gerontologist.

2010;50:363-70.

CrossRef (http://dx.doi.org/10.1093/geront/gnp139)

PubMed (http://www.ncbi.nlm.nih.gov/entrez/query.fcgi?

cmd=Retrieve\&db=PubMed\&dopt=Abstract\&list_uids=19808842)

Google Scholar (http://scholar.google.com/scholar_lookup?

title=The\%2orevised\%2odirect\%20assessment\%20of\%2ofunctional\%20status\%2ofor\%2oindependent\%20older\%20adu 370\&publication_year=2010)

37. Robnett RH, Dionne C, Jacques R, LaChance A, Mailhot M. The ManageMed Screening: an interdisciplinary tool for quickly assessing medication management skills. Clin Gerontol. 2007:30:1-22. 
CrossRef (http://dx.doi.org/10.1300/J018v30n04_01)

Google Scholar (http://scholar.google.com/scholar_lookup?

title=The\%20ManageMed\%20Screening\%3A\%20an\%2ointerdisciplinary\%2otool\%2ofor\%2oquickly\%20assessing\%2om 23\&publication_year=2007)

38. Rostgaard T. Caring for children and older people in Europe-a comparison of

European policies and practice. Policy Stud. 2002;23:51-68.

CrossRef (http://dx.doi.org/10.1080/0144287022000000082)

Google Scholar (http://scholar.google.com/scholar_lookup?

title=Caring\%20for\%20children\%20and\%20older\%2opeople\%2oin\%2oEurope\%E2\%80\%94a\%20comparison\%20of\%2 68\&publication_year=2002)

39. Stauffer Y, Spichiger E, Mischke C. Complex drug regimen in multimorbid elderly

patients after hospital discharge-a qualitative study. Pflege. 2015;28:7-18.

CrossRef (http://dx.doi.org/10.1024/1012-5302/a000400)

PubMed (http://www.ncbi.nlm.nih.gov/entrez/query.fcgi?

$\mathrm{cmd}=$ Retrieve\&db=PubMed\&dopt=Abstract\&list_uids=25631955)

Google Scholar (http://scholar.google.com/scholar_lookup?

title=Complex\%2odrug\%2oregimen\%20in\%2omultimorbid\%20elderly\%2opatients\%20after\%2ohospital\%20discharge\%

$18 \&$ publication_year $=2015$ )

40. Atkin PA, Finnegan TP, Ogle SJ, Shenfield GM. Functional ability of patients to

manage medication packaging: a survey of geriatric inpatients. Age Ageing.

1994;23:113-6.

CrossRef (http://dx.doi.org/10.1093/ageing/23.2.113)

PubMed (http://www.ncbi.nlm.nih.gov/entrez/query.fcgi?

$\mathrm{cmd}=$ Retrieve $\& \mathrm{db}=$ PubMed\&dopt=Abstract\&list_uids=8023717)

Google Scholar (http://scholar.google.com/scholar_lookup?

title=Functional\%20ability\%20of\%2opatients\%20to\%2omanage\%2omedication\%2opackaging\%3A\%20a\%20survey\%20 116\&publication_year=1994)

41. Bevil CW. Medication management in an elderly, community-based population: a

pilot project. J N Y State Nurses Assoc. 1981;12:19-29.

PubMed (http://www.ncbi.nlm.nih.gov/entrez/query.fcgi?

cmd=Retrieve\&db=PubMed\&dopt=Abstract\&list_uids=6943306)

Google Scholar (http://scholar.google.com/scholar_lookup?title=Medication\%2omanagement\%2oin\%20an\%20elderly! based\%20population\%3A\%20a\%20pilot\%20project\&author=CW.\%20Bevil\&journal=J\%20N\%20Y\%20State\%20Nurses! 29\&publication_year $=1981$ )

\section{Copyright information}

\section{(C) Springer International Publishing 2016}

\section{About this article}

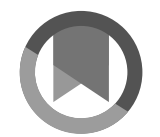

Check for updates
Print ISSN Online ISSN Publisher Name

2210-7703 2210-7711 Springer International Publishing

About this journal

$\underline{\text { Reprints and Permissions }}$ 


\section{SPRINGER NATURE}

(c) 2016 Springer International Publishing AG. Part of Springer Nature.

Not logged in · Not affiliated · 95.93.104.252 